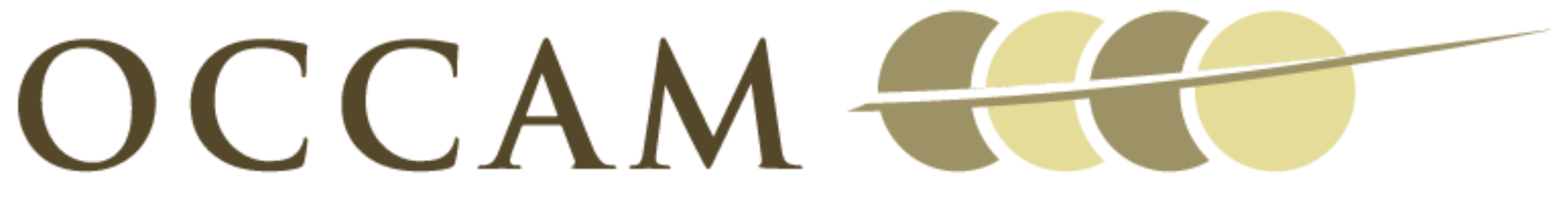

OXFORD CENTRE FOR COLLABORATIVE APPLIED MATHEMATICS

Report Number 11/29

Floating carpets and the delamination of elastic sheets

$$
\text { by }
$$

Till J. W. Wagner and Dominic Vella

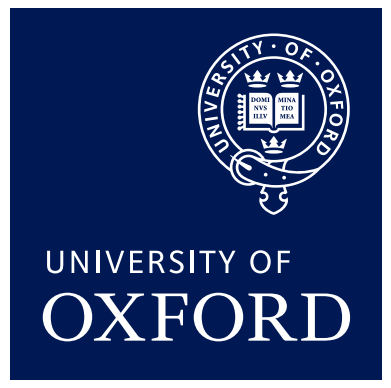

Oxford Centre for Collaborative Applied Mathematics Mathematical Institute

24 - 29 St Giles'

Oxford

OX1 3LB

England 



\title{
Floating carpets and the delamination of elastic sheets
}

\author{
Till J. W. Wagner ${ }^{1}$ and Dominic Vella ${ }^{2}$ \\ ${ }^{1}$ DAMTP, University of Cambridge, Wilberforce Rd, Cambridge, CB3 0WA, UK \\ ${ }^{2}$ OCCAM, Mathematical Institute, University of Oxford, 24-29 St Giles', Oxford, OX1 3LB, UK
}

\begin{abstract}
We investigate the deformation of a thin elastic sheet floating on a liquid surface and subject to a uniaxial compression. We show that at a critical compression the sheet delaminates from the liquid over a finite region forming a delamination 'blister'. This blistering regime adds to the wrinkling and localized folding regimes that have been studied previously. The transition from wrinkled to blistered states occurs when delamination becomes energetically favourable compared to wrinkling. We determine the initial blister size and the evolution of blister size with continuing compression before verifying our theoretical results with experiments at a macroscopic scale.
\end{abstract}

PACS numbers: 46.32.+x, 62.20.mq, 81.40.Lm

Rucks in rugs [1, 2], surfactant monolayers lining our lungs $[3,4]$, the formation of mountain ranges [5] and flexible electronic devices $[6,7]$ — all exhibit variants of Euler's classic beam buckling with two additional features: buckling takes place on a substrate and the structure that results has a length scale that is not simply the system size. In the case of a ruck in a rug, the substrate is essentially undeformable and the ruck has a finite size because of the weight of the rug. In the other cases the substrate is deformable and the degree of this deformability controls the characteristic size of the buckling pattern that results [8]. Recently, such systems have seen an explosion of interest with fundamental advances [9-12] motivated by a wealth of new applications $[13,14]$.

At its simplest, we may envisage an elastic sheet floating on the surface of a liquid and subject to uniaxial compression. It is well known that, for compressive forces above a critical value, the sheet wrinkles with a wavelength determined by a balance between resistance to bending (which penalizes short, highly curved wrinkles) and the gravitational energy of the displaced liquid (which penalizes long, large amplitude wrinkles) [9, 15]. One of the subjects of recent theoretical work has been a debate over whether localized structures emerge from this uniformly wrinkled state at a critical compression [9] or continuously [16] as a result of the finite size of the system. The debate has focussed solely on situations in which the sheet remains in contact with the liquid everywhere along its length. However, if the liquid is replaced by a soft elastic solid, it is often observed that the thin sheet delaminates from the substrate forming delamination 'blisters' [17-19]. The formation of these localized blisters is a discontinuous process occuring at a finite compression. However, a comprehensive theoretical study of when the transition occurs is complicated by the nonlinear response of the elastic substrate to deformation $[8,12,17]$. In this Letter we show that an analogous transition occurs in the case of a sheet floating at a liquid surface (demonstrating a new response of such systems) and characterize this transition theoretically.

We consider an elastic sheet of density $\rho_{s}$ and thickness

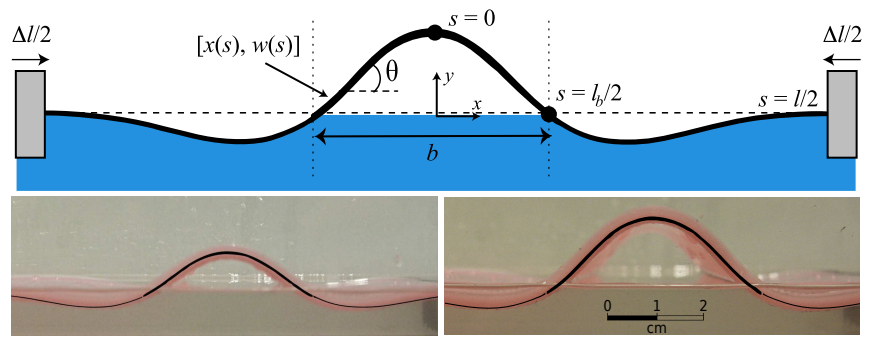

FIG. 1. (Color online) Top: Schematic diagram of a sheet floating on the surface of a liquid and subject to a uniaxial compression $\Delta l$. The compression is such that the sheet delaminates from the liquid over a horizontal distance $b$ forming a delamination 'blister'. Bottom: Experimental and theoretical profiles of a blistered sheet $(h=1.6 \mathrm{~mm})$ for $\Delta l=0.6 \mathrm{~cm}$ and $1.0 \mathrm{~cm}$, respectively.

$h$ floating on a liquid of density $\rho_{l}$ (see fig. 1). The total length of the sheet when flat is $l$ and the two ends of the sheet are brought closer together by an amount $\Delta l$. We imagine that a symmetrical delamination blister forms in the region $|s| \leq l_{b} / 2$, whose shape is to be determined along with the shape of the sheet in the region that is still wetted, $l_{b} / 2<|s|<l / 2$. We denote the shape of the sheet by $[x(s), w(s)]$ where $s$ is the arc-length (measured from the centre of the sheet) and introduce the intrinsic angle $\theta(s)$. The deformed shape of the sheet can be found by minimizing the energy of the system,

$\int_{-l / 2}^{l / 2}\left\{\frac{1}{2} D\left(\theta^{\prime}\right)^{2}+\rho_{s} g h w+\frac{1}{2} \rho_{l} g w^{2} \cos \theta \mathcal{H}\left(|s|-l_{b} / 2\right)\right\} \mathrm{d} s$,

subject to the inextensibility constraint $\int_{-l / 2}^{l / 2} \cos \theta \mathrm{d} s=$ $l-\Delta l$. In (1), the first two terms correspond to the bending and gravitational potential energies of the sheet, respectively, with $D$ the bending stiffness [15], ()$^{\prime}=\mathrm{d} / \mathrm{d} s$ and $g$ the acceleration due to gravity. The third term corresponds to the gravitational potential energy of the displaced liquid and the Heaviside step-function $\mathcal{H}(x)$ ensures that liquid is only displaced in the region $l_{b} / 2<$ 
$|s|<l / 2$. Performing this minimization, we find

$$
\left(\frac{D \theta^{\prime \prime}+T \sin \theta+\rho_{s} g h s \cos \theta}{\cos \theta}\right)^{\prime}=\left\{\begin{array}{l}
0, \quad|s| \leq l_{b} / 2 \\
-\frac{\rho_{l} g}{2 w^{\prime}}\left(\frac{w^{2}}{\cos \theta}\right)^{\prime} \\
l_{b} / 2 \leq|s| \leq l / 2
\end{array}\right.
$$

with $x^{\prime}=\sin \theta$ and $w^{\prime}=\cos \theta$ where $T$ is the compressive force applied. ( $T$ emerges as the Lagrange multiplier enforcing the inextensibility constraint.) Note that in the blistered region $|s| \leq l_{b} / 2$, (2) recovers the equation for a 'ruck in a rug' given previously $[1,2,20]$.

The balance between bending stiffness and hydrostatic pressure leads to a natural lengthscale for non-blistered floating sheets, $\ell_{w} \equiv\left(D / \rho_{l} g\right)^{1 / 4}$. Physically, the length $\ell_{w}$ is related to the wavelength of the wrinkling instability that occurs when a floating sheet is compressed [15]. We use $\ell_{w}$ to non-dimensionalize lengths so that $S=s / \ell_{w}$, $X=x / \ell_{w}$ etc. However, we let $W=\left(w-w_{\infty}\right) / \ell_{w}$ where $w_{\infty} \equiv h\left(1 / 2-\rho_{s} / \rho_{l}\right)$ is the depth of the centerline below the liquid when freely floating; $W$ measures deflections of the sheet centerline from its equilibrium floating position. We also introduce a dimensionless compressive force $\tau=$ $T /\left(D \rho_{l} g\right)^{1 / 2}$ and the draft of the sheet $\delta \equiv \rho_{s} h / \rho_{l} \ell_{w}$.

The dimensionless version of $(2)$ is to be solved subject to the symmetry conditions $\theta(0)=0, X(0)=0$ along with clamped conditions at the edges of the sheet, $W(L / 2)=W^{\prime}(L / 2)=0$, and an imposed displacement $X(L / 2)=(L-\Delta L) / 2$. An additional condition arises from the bottom surface of the sheet touching the liquid at its natural level when $S=L_{b} / 2$ so that $W\left(L_{b}^{-} / 2\right)=$ $W\left(L_{b}^{+} / 2\right)=\delta$. Finally, we have continuity conditions at the edge of the blister $[\theta]_{-}^{+}=\left[\theta^{\prime}\right]_{-}^{+}=\left[\theta^{\prime \prime}\right]_{-}^{+}=0$.

Equation (2) may be solved numerically using, for example, the MATLAB routine bvp4c. However, a great deal of understanding can be obtained by considering the linearized theory (valid for small deformations $\theta \ll 1$ ) and letting $L \rightarrow \infty$. In this limit, $\delta$ can be rescaled out of the problem by letting $\omega=W / \delta$ yielding the analytical solution:

$$
\frac{W}{\delta}=\left\{\begin{array}{cl}
\beta_{1}\left(\cos \tau^{1 / 2} X-\cos \frac{\tau^{1 / 2} B}{2}\right)+ & \left(\frac{B^{2}-4 X^{2}}{8 \tau}+1\right), \\
& (0<X<B / 2) \\
e^{-\lambda \xi}\left\{\cos \mu \xi+\beta_{2} \sin \mu \xi\right\}, & (X>B / 2)
\end{array}\right.
$$

where $\xi=X-B / 2, \quad \lambda=(2-\tau)^{1 / 2} / 2, \quad \mu=(2+\tau)^{1 / 2} / 2$, the constants $\beta_{1,2}$ are given by

$$
\begin{aligned}
& \beta_{1}=\tau^{-3 / 2}[2 \lambda \tau+B(\tau-1) / 2] \csc \frac{B \tau^{1 / 2}}{2}, \\
& \beta_{2}=-\frac{B}{2 \mu}-\frac{\lambda}{\mu},
\end{aligned}
$$

and the compressive force $\tau$ is found as a function of the blister size $B$ from

$$
\begin{aligned}
1 & +\tau-\tau^{2}+\frac{B \tau}{2}(2-\tau)^{1 / 2} \\
& =\left[1-\tau+\tau^{3 / 2}(2-\tau)^{1 / 2}\right] \frac{B \tau^{1 / 2}}{2} \cot \frac{B \tau^{1 / 2}}{2} .
\end{aligned}
$$

In the limit of large blisters, $B \gg 1$, we find $\tau \approx 4 \alpha^{2} / B^{2}$ where $\alpha \approx 4.493$ is the smallest positive solution of $x=$ $\tan x$. The limit $B \gg 1$ thus reduces to the buckling of a heavy elastica $[1,2,20]$, or 'ruck in a rug', because the external compression accommodated in the adhered region is small compared to that within the blister.

We anticipate that the blistering state should be observed when it is energetically favourable in comparison to the alternative, i.e. wrinkled, state. We must therefore determine the total energy of the blistered state. For convenience, we measure the dimensionless energy $\mathcal{U} \equiv U /\left(D / \ell_{w}\right)$ relative to the dimensionless energy of an undeformed floating sheet. This requires the subtraction of the isostatic energy from that given in (1). We must add to this energy the surface energy penalty associated with delamination. Assuming that the blistered part remains wet, as has been reported in related problems [21], this surface energy is $U_{s}^{(b)}=\gamma_{l v}\left(b+l_{b}\right)$ where $\gamma_{l v}$ is the energy per unit area associated with the liquid-vapour interface. In dimensionless terms, this reads

$$
\mathcal{U}_{s}^{(b)}=\Gamma\left(B+L_{b}\right)
$$

where $\Gamma \equiv \gamma_{l v} \ell_{w}^{2} / D$ is the dimensionless strength of surface tension. The energy in the wrinkled state has previously been shown to be [16],

$$
\mathcal{U}^{(w)}=2 \Delta L-\Delta L^{2} / L,
$$

to second order in $\Delta L$. However, in the limit $L \rightarrow \infty$ this becomes $\mathcal{U}^{(w)}=2 \Delta L$. Blistering is therefore energetically favourable compared to wrinkling with the same compression, $\Delta L$, whenever $\mathcal{U}_{e+g}^{(b)}+U_{s}^{(b)}<\mathcal{U}^{(w)}$ (for a given compression, $\Delta L, B$ can be determined by computing $\left.B=2 X\left(L_{b} / 2\right)\right)$. For given values of $\delta$ and $\Gamma$ the critical compression or, equivalently, critical blister size $B=B_{c}$ at which blistering occurs can be computed numerically. However, it is instructive to consider the limit of small deformations, where the blister profile is given by (3) and $B \approx L_{b}$. In this regime it is a simple matter to show that $\mathcal{U}_{e+g}^{(b)}=\delta^{2} f(B)$ and that $\Delta L=\delta^{2} g(B)$ for some functions $f$ and $g$, which can be computed numerically. Crucially, however, the quadratic dependence of each of these terms on $\delta$ shows that the inequality for blistering may be written

$$
2 \frac{\Gamma}{\delta^{2}} B+f(B)<2 g(B)
$$

and hence we see that, within the linear theory at least, the critical blister size $B_{c}=B_{c}\left(\Gamma / \delta^{2}\right)$. 


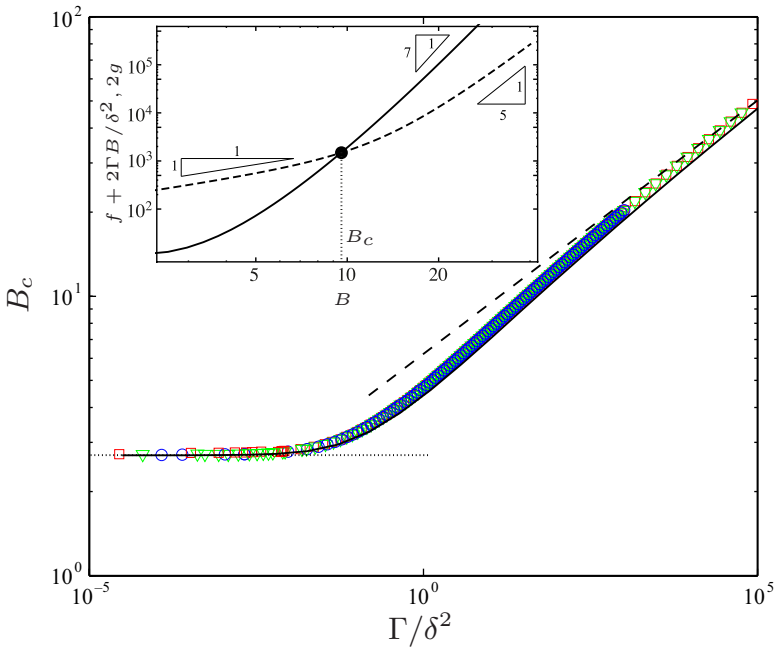

FIG. 2. (Color online) Main figure: Critical blister size at onset $B_{c}$ as a function of $\Gamma / \delta^{2}$. Results from numerical solutions of (2) are shown as points: $\delta=10^{-5}(\bigcirc), \delta=10^{-4}$ $(\square)$ and $\delta=10^{-3}(\nabla)$ all with $L=500$. The results of the linear theory (solid curve) give rise to the asymptotic results (6). Inset: The functions in the inequality $(5)$ for $\Gamma / \delta^{2}=50$ : $f(B)+2 \Gamma B / \delta^{2}$ (dashed curve) and $2 g(B)$ (solid curve). Blistering is energetically favourable for $B>B_{c}$. The scaling behaviors of these functions in various limits are also illustrated (see text).

The inset of fig. 2 shows the behavior of the left hand side of the inequality (5) for $\Gamma / \delta^{2}=50$, along with the behavior of the right hand side of (5). This demonstrates that for sufficiently large $B$ (corresponding to sufficiently large compressions $\Delta L$ ) the formation of a delamination blister becomes energetically favourable to wrinkling. Altering the value of $\Gamma / \delta^{2}$ simply corresponds to shifting the dashed curve in this inset vertically, altering the observed blister size at onset. The main body of fig. 2 shows the dependence of $B_{c}$ on $\Gamma / \delta^{2}$ obtained from both numerical simulations and the linear theory.

For $B \gg 1$ the ruck in a rug solution $[1,2]$ gives $f(B) \sim$ $0.0018057 B^{5}$ and $g(B) \sim 0.00001597 B^{7}$ (these prefactors may be expressed as analytical, but cumbersome, functions of $\alpha$ ). Substituting these expressions into (5) we find that $B \gg 1$ only if $\Gamma / \delta^{2} \gg 1$ and blistering is energetically preferable for $\Delta L>6.302 \Gamma^{7 / 6} \delta^{-1 / 3}$. On the other hand, for $\Gamma / \delta^{2} \ll 1$, (5) reduces to $f(B)<2 g(B)$ and it is clear that we require $B>B_{c}$ with $B_{c}=O(1)$. A numerical computation reveals that this requires a critical compression $\Delta L_{c} \approx 6.597 \delta^{2}$. These results correspond to critical blister sizes

$$
B_{c} \approx\left\{\begin{array}{l}
2.627, \quad \Gamma / \delta^{2} \ll 1 \\
6.302\left(\Gamma / \delta^{2}\right)^{1 / 6}, \quad \Gamma / \delta^{2} \gg 1
\end{array}\right.
$$

We note from fig. 2 that these asymptotic limits are recovered by both the linear and nonlinear computations.

The predictions of the preceding theoretical analysis were tested using macroscopic experiments with rubber sheets of dimensions $40 \mathrm{~cm} \times 10 \mathrm{~cm}$ and different thicknesses in the interval $0.6 \mathrm{~mm} \lesssim h \lesssim 1.6 \mathrm{~mm}$. The bending rigidity $D=E h^{3} / 12\left(1-\nu^{2}\right)$ was measured by means of the elastic loop test [22] and found to be consistent with a Young's modulus $E=220 \pm 10 \mathrm{kPa}$ and Poisson ratio $\nu \approx 0.5$ [19]. Experiments were performed using potassium carbonate as a liquid layer of depth $>3 \mathrm{~cm}$ $\left(\mathrm{K}_{2} \mathrm{CO}_{3}, \rho_{l}=1520 \mathrm{kgm}^{-3}, \gamma_{l v}=102 \mathrm{mNm}^{-1}\right)$. To impose clamped boundary conditions the ends of the rubber sheets were taped to perspex blocks. These blocks were then moved together quasi-statically, compressing the sheet. As expected from the preceding calculations, a delamination blister forms spontaneously at a critical compression. Typical blister shapes are shown in fig. 1 and compared to those predicted theoretically [23]. We observe good agreement between experiment and theory in these instances.

Fig. 3 shows measured values of $b_{c}$ for different sheet thicknesses in comparison with that predicted from numerical simulations [24]. This demonstrates good agreement with experiment. Experimental and numerical values of the size of the blister for varying compressions $\Delta L$ are shown in the inset of fig. 3 . We note that in this situation experiments in both compression and relaxation are repeatable and reproduce the same critical compression for the appearance/disappearance of the blister - there is no observable hysteresis, unlike that observed in related problems [19]. Moreover, the nonlinear simulations explain a feature of the experiment that is not predicted by the simple linear theory: we observe a non-monotonic relationship between the blister size $B$ and the compression $\Delta L$ with an intermediate maximum value of $B$.

We have introduced a new type of deformation for uniaxially compressed sheets: over a region of well-defined size the sheet deadheres from the liquid, forming a delamination blister. We showed that this 'blistering' is a discontinuous process that occurs at a well-defined compression $\Delta L_{c}$ and is manifested by the appearance of a blister with a well-defined size $B_{c}$. This transition occurs when the energy required to accommodate a given displacement by wrinkling exceeds that required to accommodate the same displacement by blistering. We have shown that, within the linear theory, the blister size at onset, $B_{c}$, is a function of the rescaled surface energy $\Gamma / \delta^{2}$ only and characterized this dependence with scaling laws in the limits $\Gamma / \delta^{2} \ll 1$ and $\Gamma / \delta^{2} \gg 1$. That the blister size observed at onset increases with the surface energy $\gamma_{l v}$ is both surprising and in contrast to what has been found for the case of a sheet delaminating from a soft elastic substrate [19]. However, this behavior is simple to understand qualitatively: for stronger adhesion a greater compression needs to be applied to cause delamination (as is also the case for a sheet delaminating from a soft substrate [19]). In the case of a floating sheet this causes a larger blister to be formed. Nevertheless, we note that the observed dependence is relatively weak, 


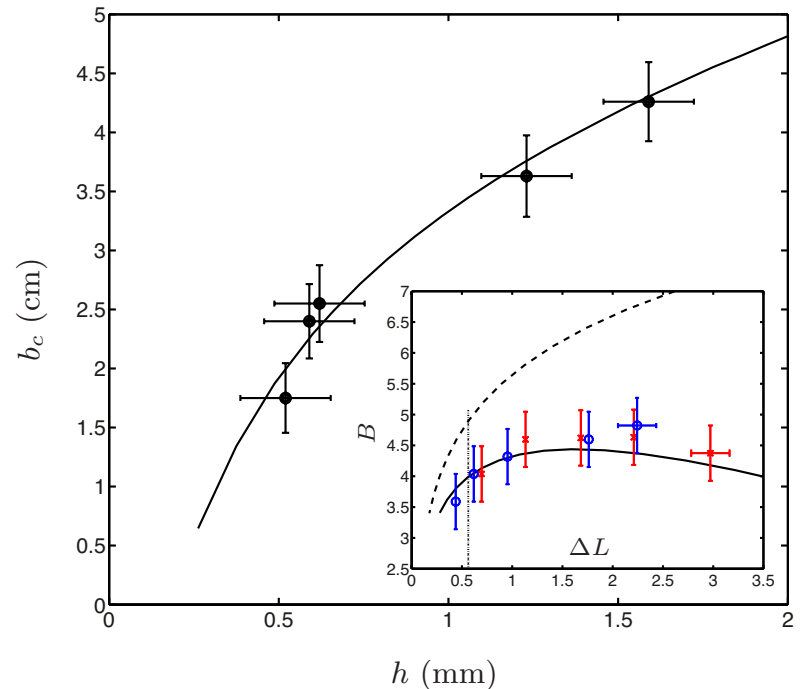

FIG. 3. (Color online) Main figure: Blister size at onset, $b_{c}$, for different sheet thicknesses, $h$, measured experimentally (points) and compared to the prediction from numerical computations (solid curve). Inset: The evolution of $B$ with varying compression $\Delta L$. Experimental results are shown for compression $(x)$ and relaxation $(\bigcirc)$ with the results of the nonlinear theory (solid curve) and the linear result $B \approx 4.844 \Delta L^{1 / 7}[1]$ (dashed curve). The vertical dotted line represents the predicted onset of blistering, $\Delta L_{c}$.

$b_{c} \sim \gamma_{l v}^{1 / 6}$

The theory developed in this Letter is a first step towards understanding delamination more generally. The restoring force that is provided by hydrostatic pressure is relatively simple to model and is analogous to the response of thin elastic substrates, or Winkler foundations. Hence the delamination of thin films from a thin elastic layer of thickness $h_{l}$ and with Lamé constants $\lambda$ and $\mu$ should be described by the theory presented here with $\rho_{l} g$ replaced by a stiffness $(\lambda+2 \mu) / h_{l}[25]$. For thick substrates more detailed analysis is required but may yield new insights beyond those obtained from fully numerical approaches such as the finite element method $[17,26]$.

It remains to understand fully the regimes in which the different scenarios of wrinkling, blistering and folding are found. Here we obtained analytical results in the limit of infinite system size, $L \rightarrow \infty$, in which case blistering is ultimately energetically more favourable than wrinkling. However, with a finite system size the energy of the wrinkled state includes a non-linear term that acts to decrease the elastic energy and hence may suppress the blistering instability. This is likely to be particularly pertinent in the limit of large surface tension, $\Gamma / \delta^{2} \gg 1$. For example, using the relevant values for thin polyester films floating on water [9] we find that $\Gamma / \delta^{2} \approx 4 \times 10^{4}$ and $\ell_{w}=2.5 \mathrm{~mm}$. According to (6) blisters will only occur in these systems if the compression $\Delta l>10 \mathrm{~cm}$, which is significantly larger than the compressions re- quired to observe folding in these systems [9, 16]. However, in less confined systems subject to large stresses - e.g. floating sea ice sheets - the delamination presented here might naturally be observed. Indeed, Arctic explorer Julius Payer offers a tantalizing allusion to this, writing in his diary: '... immeasurable pressure causes [the ice floes] to bend like arcs, yes, in blisters they rise, a terrifying sign of the elasticity of the ice ...' [27].

This publication is based on work supported in part by Award No. KUK-C1-013-04, made by King Abdullah University of Science and Technology (KAUST). TJWW is supported by EPSRC. We thank R. Sayag and D. Page-Croft for technical assistance with experiments.

[1] D. Vella et al., Phys. Rev. Lett. 103, 174301 (2009).

[2] J. M. Kolinski, P. Aussillous, and L. Mahadevan, Phys. Rev. Lett. 103, 174302 (2009).

[3] M. M. Lipp et al., Phys. Rev. Lett. 81, 1650 (1998).

[4] H. Diamant et al., Phys. Rev. E 63, 061602 (2001).

[5] H. Ramberg and O. Stephansson, Tectonophysics 1, 101 (1964).

[6] J. A. Rogers et al., Science 327, 1603 (2010).

[7] S. Wang et al., Soft Matter 6, 5757 (2010).

[8] J. Groenewold, Physica A 298, 32 (2001).

[9] L. Pocivavsek et al., Science 320, 912 (2008).

[10] P. M. Reis et al., Phys. Rev. Lett. 103, 045501 (2009).

[11] B. D. Leahy et al., Phys. Rev. Lett. 105, 058301 (2010).

[12] F. Brau et al., Nature Phys. 7, 56 (2011).

[13] N. Bowden et al., Nature 393, 146 (1998).

[14] C. M. Stafford et al., Nature Mater. 3, 545 (2004).

[15] L. D. Landau and E. M. Lifschitz, The Theory of Elasticity (Pergamon, 1970).

[16] H. Diamant and T. A. Witten, arxiv (2010).

[17] G. Parry et al., Acta Mater. 53, 441 (2005).

[18] H. Mei et al., Appl. Phys. Lett. 90, 151902 (2007).

[19] D. Vella et al., Proc. Natl. Acad. Sci. USA 106, 10901 (2009).

[20] C. Y. Wang, Int. J. Mech. Sci 28, 549 (1986).

[21] J. Chopin et al., Proc. R. Soc. A 464, 2887 (2008).

[22] I. M. Stuart, Brit. J. Appl. Phys. 17, 1215 (1966).

[23] In these comparisons, the fully nonlinear beam equation (2) is used to model the deformation of the blistered part of the sheet, although the adhered part is modelled using the linear theory since it only deforms slightly.

[24] Errorbars arise from variations in sheet thicknesses and the liquid meniscus at the tank wall. Horizontal errorbars are only representative and illustrate setup inaccuracies (error in clamping conditions, non-uniformity in the plane of the liquid surface) as well as an error in measurements of the critical compression from the dynamic nature of the experiment.

[25] J. M. Skotheim and L. Mahadevan, Phys. Rev. Lett. 92, 245509 (2004).

[26] H.-H. Yu and J. W. Hutchinson, Int. J. Fracture 113, 39 (2002).

[27] J. von Payer, The Austro-Hungarian North Pole Expedition of 1869 to 1874 (Vienna, 1876). 



\section{RECENT REPORTS}

06/11 A numerical methodology for the Painleve equations

Fornberg

Weideman

07/11 Strong stability preserving two-step Runke-Kutta methods

Ketcheson

Gotlieb

MacDonald

08/11 Hysteresis and Post Walrasian Economics

Cross

McNamara

Kalachev

Pokrovskii

09/11 A locally adaptive time-stepping algorithm for petroleum reservoir simulations

McNamara

Bowen

Dellar

10/11 On the predictions and limitations of the BeckerDoring model for reaction kinetics in micellar surfactant solutions

Griffiths

Bain

Breward

Colegate

Howell

Waters

11/11 Dynamics of the Tear Film Braun

12/11 The infuence of receptor-mediated interactions on reaction- Klikaa diffusion mechanisms of cellular self-organisation Baker

Headon

Gaffney

13/11 Quasi-steady state analysis of two-dimensional random intermit- Bressloff tent search processes $\quad$ Newby

14/11 A Constrained Approach to Multiscale Stochastic Simulation of Cotter Chemically Reacting Systems

Zygalakis

Kevrekidis

Erban

15/11 The Two Regime Method for optimizing stochastic reaction- Flegg diffusion simulations

Chapman

Erban

16/11 Recombination via tail states in polythiophene:fullerene solar cells Kirchartz

Pieters

Kirkpatrick

Rau

Nelson

17/11 Energy versus electron transfer in organic solar cells: a comparison Soon of the photophysics of two indenofluorene: fullerene blend films Clarke

Zhang

Agostinelli

Kirkpatrick

Dyer-Smith

McCulloch

Nelson

Durrant 
22/11 A novel model for one-dimensional morphoelasticity. Part II: Ap- Hall plication to the contraction of fibroblast-populated collagen lat- Menon tices

23/11 Positive or negative Poynting efect? The role of adscititious in-

Mihai equalities in hyperelastic materials

Goriely

McCue

McElwain

24/11 On approaches to modelling lattice dislocations

Hall

Markenscoff

25/11 Nonlinear waves in heterogeneous elastic rods via homogenization

de Luna

Emptage

Goriely

Bressloff

26/11 Synaptic bistability due to nucleation and evaporation of receptor

Burlakov clusters

Duričković

Goriely

27/11 Particle trapping and banding in rapid solidification

Elliot

Peppin

28/11 Growth of confined cancer spheroids: a combined experimental

Loessner and mathematical modelling approach

Flegg

Byrne

Hall

Moroney

Clements

McElwain

Hutmacher

Copies of these, and any other OCCAM reports can be obtained from:

Oxford Centre for Collaborative Applied Mathematics Mathematical Institute

24 - 29 St Giles'

Oxford

OX1 3LB

England

www.maths.ox.ac.uk/occam 